\title{
Le rôle des réseaux d'acteurs dans l'innovation publique complexe : le cas du vote électronique dans le canton de Genève
}

\author{
> Owen Boukamel \\ Institut de Hautes Études en Administration Publique (IDHEAP), \\ Université de Lausanne
}

Bâtiment IDHEAP, UNIL, CH-1015 Lausanne, Suisse

\section{Résumé}

Les réseaux d'acteurs jouent un rôle essentiel dans le déroulement des innovations. Or, dans le secteur public, les innovations sont souvent empreintes d'une grande complexité. Ce caractère complexe modifie substantiellement les processus d'innovation, leurs freins, leurs leviers et leurs résultats, et donc le rôle des réseaux d'acteurs à chaque phase du processus. Ces changements sont illustrés à travers une étude de cas exemplaire portant sur le vote électronique dans le canton de Genève, projet éminemment complexe.

๑) 2017 IDMP/Lavoisier SAS. Tous droits réservés

Mots clés : innovation publique, réseaux d'acteurs, complexité.

\section{Abstract}

The role of networks of actors on complex innovation in the public sector: the case of e-voting in the canton of Geneva. Networks of actors play an essential role on innovation processes. In the public sector, innovations are often highly complex. This complex nature substantially affects innovation processes, their barriers, their drivers and their outcomes. Complexity also subverts the role of networks in each phase of innovation processes. These changes are illustrated through an exemplary case study of the complex evoting project, in the canton of Geneva.

(c) 2017 IDMP/Lavoisier SAS. Tous droits réservés

Keywords : public sector innovation, networks of actors, complexity.

*Auteur correspondant : owen.boukamel@unil.ch

doi :10.3166/pmp.34. 2017.0010 @ 2017 IDMP/Lavoisier SAS. Tous droits réservés 


\section{Introduction}

Le rôle de l'État vis-à-vis de l'innovation a longtemps été celui de créer les conditions-cadres pour que le secteur privé, lui, puisse innover. Progressivement, les modèles traditionnels de l'administration se sont émiettés : le New Public Management (Pollitt et Bouckaert, 2011) a cédé la place à d'autres paradigmes dans lesquels la coopération supplante la compétition, les liens entre les organisations s'horizontalisent (Kinder, 2013), les politiciens et managers deviennent des relais entre les acteurs, et les citoyens, eux, des co-créateurs de leurs propres services publics (Christensen et Lægreid, 2016 : 294). L'administration a dû, progressivement, devenir actrice de l'innovation. D'autres éléments, telles que les réformes budgétaires, obligent pour de bon l'administration publique à faire preuve de créativité et d'agilité.

Ce phénomène d'innovation publique est désormais un thème de recherche prégnant (Boukamel et Emery, 2017; Bekkers, Tummers, et Vooberg, 2013; Kattel, 2015 ; Osborne et Brown, 2011). Cette jeune littérature s'accorde sur le fait que certaines particularités publiques comme l'absence de concurrence ou la « mission » de service public, rendent les phénomènes d'innovation publics bien singuliers (Halvorsen, Hauknes, Miles et Rannveig, 2005 ; Kay et Goldspink, 2016). En plus d'être particulièrement ouvertes vis-à-vis de leur environnement (Walker, 2008), ainsi que dans leur manière de se diffuser (Chesbrough, 2003), les innovations publiques seraient souvent multidimensionnelles, c'est-à-dire qu'elles impliqueraient des changements de plusieurs natures (Torugsa et Arundel, 2016). À cela s'ajouterait la propension des innovations publiques à mobiliser toute une écologie d'acteurs éclatés (Dougherty et Dunne, 2011; Touati, Denis, Grenier, et Smits, 2016) : départements, services ou entités publiques, a priori peu enclins à travailler ensemble, fortement institutionnalisés, ou du moins, dont le fonctionnement routinier rend la coopération exceptionnelle (Michaux, 2010; Touati et al., 2016). Tous ces éléments conduisent à penser que les innovations publiques seraient souvent complexes.

C'est précisément vers ces processus d'interactions complexes de l'innovation publique que les chercheurs se tournent depuis peu, montrant, entre autres, l'importance de cette complexité sur la marche de l'innovation, sur ses freins et leviers, sur l'incertitude qui l'entoure ainsi que sur la versatilité de son déroulement.

Dès lors, tout laisse à penser que l'espace social -décrit comme un moteur de l'innovation- n'est plus identifiable au sein d'une organisation précise : il est atomisé (Kinder, 2013), momentané, parfois intangible, et d'ailleurs souvent informel. En parallèle des structures officielles créées pour les besoins du projet, des réseaux d'acteurs s'activent ou se développent. Moins visibles, ces réseaux ont déjà été identifiés comme étant des accélérateurs de l'innovation, d'abord dans la littérature sur le secteur privé (Fu, Revilla Diez, et Schiller, 2013), puis publique (Alexander, Lewis, et Considine, 2011 ; Lewis, Considine, et Alexander, 2011; Lewis et Ricard, 2014). Mais que savons-nous du rôle de ces réseaux d'acteurs dans le cadre d'innovations particulièrement complexes, sachant que la complexité modifie les leviers traditionnels de l'innovation? En d'autres termes, quel est le rôle des réseaux dans les innovations complexes, multidimensionnelles et multi-acteurs au sein des administrations publiques ? Tout laisse à penser que le rôle des réseaux d'acteurs est, dans la gestion de la complexité, encore plus important que pour les innovations moins complexes où les canaux formels pourraient suffire. 
L'objectif de cette recherche est d'explorer, à travers la littérature et l'étude d'un cas illustratif, le rôle que jouent les réseaux d'acteurs sur les processus d'innovation publique quand celle-ci est particulièrement complexe. Cet article s'inscrit dans la démarche d'autonomisation de l'étude de l'innovation publique vis-à-vis de la recherche sur le secteur privé.

\section{Revue de littérature}

Nous aborderons tout d'abord le concept d'innovation publique complexe. Nous examinerons ce qu'est la complexité, puis nous verrons dans un deuxième temps, de quelle manière celle-ci interfère avec les processus d'innovations. Nous nous intéresserons ensuite aux réseaux d'acteurs comme espace de l'innovation de manière générale, puis, -dans la cinquième partie-, en tant qu'espace de l'innovation publique. En pénétrant ce lien qui unit réseaux et processus d'innovation, nous serons en mesure d'entrevoir les potentiels changements provoqués par la complexité.

\subsection{L'innovation publique}

Construite à partir de l'importation « brute » dans le public des travaux sur l'innovation des entreprises privées (Salge et Vera, 2012), la littérature publique s'est progressivement émancipée et s'affirme depuis peu comme un champ d'étude à part entière (Kattel, Cepilovs, Kalvet, Lember, et Tonurist, 2016). Pour cette raison, certains auteurs déplorent encore le manque de vision intégrée (De Vries et al., 2015 ; Osborne et Brown, 2011). À ce jour, il n'y a donc toujours pas de définition robuste de l'innovation publique (Daglio, Gerson, et Kitchen, 2015; De Vries et al., 2016).

Malgré tout, un consensus se forme autour de l'idée que l'innovation dans le secteur public serait très différente de celle du secteur privé. Dans le secteur public, les frontières organisationnelles sont poreuses, les organisations doivent collaborer et non se concurrencer, les processus doivent être particulièrement transparents, etc. Toutes ces particularités font que l'innovation publique ne naît presque jamais d'un service $R \& D$ avant de se diffuser unilatéralement au reste de l'organisation, à l'image du secteur privé. « Le contenu, le déroulement et les résultats du processus d'innovation [sont] le résultat d'interactions complexes entre des antécédents, des ressources et des acteurs intra-organisationnels et des antécédents, des ressources et des acteurs externes. » (De Vries et al., 2016 : 147).

Malgré la relative jeunesse de la littérature sur l'innovation publique, Eggers et Singh (2009) puis Sørensen et Torfing (2011) ont identifié quatre phases dans ces processus spécifiques.

1.L'émergence d'idées. C'est la première phase du cycle d'innovation. Cette phase désigne le développement des idées sur la base de l'identification des problèmes et des opportunités, et à partir des buts et de la culture de l'organisation.

2.La sélection des idées. Après une phase d'émergence d'idées, l'organisation doit arbitrer les idées qui méritent d'être poursuivies. Dans cette phase, l'idéalisme est soumis au pragmatisme : on étudie la faisabilité des idées et leur potentielle acceptation par les principaux acteurs. Les processus de négociation, de compromis et de résolution des conflits sont des éléments clés de cette phase. 
3. La mise en æuvre. Cette phase comprend la traduction des idées en nouvelles procédures, pratiques, services, etc. Elle implique un changement des habitudes de travail et des routines et donc des résistances potentiellement fortes. Elle doit donc se baser sur un leadership solide, une appropriation de l'innovation par les acteurs mais aussi des aspects incitatifs. La mise en œuvre est souvent pleine d'incertitude.

4.La diffusion des nouvelles pratiques. On observe dans cette phase la diffusion de l'innovation à toute l'organisation ou même à d'autres entités extérieures. Son succès repose sur la perception des bénéfices obtenus jusqu'à présent par la mise en œuvre de l'innovation, sur une certaine culture d'ouverture aux idées nouvelles et sur la connexion entre les acteurs innovateurs et les adopteurs potentiels.

Ce phasage est très utile - il nous servira d'ailleurs de grille d'analyse -, mais ne doit pas être vu comme un enchaînement linéaire. En fait ces phases se chevauchent, se combinent les unes aux autres et se répètent parfois. Une des causes de cette versatilité des processus serait la complexité de l'innovation.

\subsection{La complexité des innovations publiques}

C'est en voulant adapter la typologie schumpetérienne des innovations (produits, procédés, commercialisation, sources d'approvisionnement, organisation (OECD/Eurostat, 2005)) au secteur public, que l'on a fait le constat de la relative complexité des innovations publiques.

Les premières typologisations publiques ont conduit à l'établissement de catégories assez proches de celles du privé. On y retrouve les innovations de service, de processus et d'organisation, à l'exception de deux nouveaux types : les innovations de politiques publiques (développer des politiques publiques d'un nouveau genre) et les innovations conceptuelles (qui désignent l'adoption de nouvelles idées et concepts par des administrations qui en font un fil rouge de leur action) (Torugsa et Arundel, 2016; Windrum, 2008). Même si cette catégorisation est plus adaptée au secteur public, cette typologie est difficilement observable empiriquement. En effet, la mise en place d'un guichet unique pour les démarches administratives, par exemple, implique un nouveau service aux yeux des usagers, mais aussi une réorganisation des structures organisationnelles et des procédures en interne. En fait, les chercheurs ont constaté que les innovations publiques n'impliquaient pas uniquement un service ou une structure organisationnelle, pour ne citer qu'eux, mais bien souvent plusieurs de ces aspects à la fois (Goffin et Mitchell, 2010; Torugsa et Arundel, 2016). Ce phénomène, baptisé multidimensionalité, contribue à la complexité des innovations publiques (Torugsa et Arundel, 2016).

En plus d'être multidimensionnelles, les innovations publiques peuvent être transversales et multi-acteurs. Elles peuvent concerner dans leurs différentes phases, une multitude d'institutions, d'organisations (Armbrustera, Bikfalvib, Kinkela, et Laya, 2008; Camisón et Villar-López, 2014) et d'acteurs, plus ou moins consultés ou impliqués, parfois même franchement co-constructeurs de l'innovation. La transversalité (l'implication d'administrations, d'institutions, d'organisations ou d'unités de travail extérieures au projet) est nécessaire puisque les attentes des citoyens sont le plus souvent multiples. Pour reprendre un exemple de Kinder (2003), une personne déménageant dans une commune voisine sollicitera tant l'administration fiscale, que l'école, la clinique ou le fournisseur d'énergie. Illustrant ce besoin de transversalité, on observe des dispositifs de guichets uniques éclore çà et là. Pourtant cette transversalité est souvent extrêmement difficile à atteindre tant les frontières institutionnelles 
peuvent être fortes (Michaux, 2010). Par ailleurs, des acteurs non-institutionnels, comme des citoyens ou des entrepreneurs, peuvent être intégrés aux projets. Dans ces cas-là, il ne s'agit pas de transversalité mais de caractère multi-acteurs au sens plus large. Ici «l'espace » de l'innovation est éclaté : on ne parle plus d'organisation mais de réseaux complexes (Rhodes, 2013), de systèmes d'innovation (Kinder, 2013) ou encore d'écologie d'acteurs (Dougherty et Dunne, 2011; Touati et al., 2016), qui s'ajoutent à la complexité.

Il n'existe pas encore de définition précise de la complexité d'une innovation. À la lumière des éléments soulevés dans cette partie, la complexité d'une innovation publique pourrait être définie en fonction de ce qu'elle implique (multi-dimensionnalité) et de ceux qu'elle implique (transversalité et multi-acteurs), dans des milieux fortement institutionnalisés. Plus l'innovation conduit à des changements nouveaux dans de nombreux domaines, mobilisant des réseaux d'acteurs larges et éclatés, plus elle sera complexe. Nous retiendrons cette approche dans cet article.

Comme nous allons le voir, cette complexité a de nombreuses incidences sur ce que l'on pourrait nommer l'espace-temps des processus d'innovation.

\subsection{Les effets de la complexité sur les processus d'innovation}

Plusieurs études montrent que la complexité d'une innovation interfère sur son déroulement. Dans le secteur privé, la complexité de l'innovation ferait substantiellement osciller ses processus, amplifierait l'enchevêtrement de ses phases, rendant le projet plus incertain, moins intelligible pour les acteurs et son pilotage moins contrôlable (Gopalakrishnan et Bierly, 2001 ; Gopalakrishnan et Damanpour, 1994 ; Pelz, 1985).

Dans le secteur public, le constat est le même : les innovations complexes ne sont pas linéaires, leurs processus bégaient (Kinder, 2013 : 319). Torugsa et Arundel (2016) montrent que la complexité agit non seulement sur le déroulement de l'innovation publique, -à l'instar des travaux antérieurs sur le secteur privé-, mais aussi sur ses freins et ses leviers. Dans les cas d'innovations complexes, les freins sont exacerbés. Les leviers, eux, sont différents : les innovations complexes requièrent -pour leur bon déroulement-, un cheminement davantage ascendant (bottom-up) ainsi qu'un plus grand nombre de sources d'idées. Les auteurs montrent aussi qu'une configuration du travail plus décentralisée et autonomisée serait plus favorable aux innovations complexes, car bénéfique pour la créativité individuelle et collective.

Pour Kinder (2013), qui s’intéresse plus particulièrement à la transversalité, la « bonne » gestion de la complexité passe par la capacité de l'administration à régulièrement réviser ses processus opérationnels et à s'appuyer sur des équipes multidisciplinaires, créatives (ce qui rejoint l'idée de Torugsa et Arundel (2016)) et capables de briser les frontières entre les espaces fortement institutionnalisés (argument que l'on retrouve chez Zietsma et Lawrence (2010)). La transversalité exige également que la circulation de l'information soit efficace dans les deux sens (descendante et ascendante), que les ressources soient mises en commun, que des acteurs externes intègrent la conception du projet, et enfin, que des dispositifs entretiennent une certaine culture de l'innovation.

En résumé, une innovation publique complexe se distingue d'une innovation simple de trois façons. Tout d'abord, la complexité bouleverse ce que nous nommerons le temps de l'innovation : les différentes phases s'enchevêtrent et le déroulement est irrégulier. Ensuite, la complexité amplifie les freins, et modifie les leviers associés à chacune des phases de 
l'innovation. Si par exemple, l'implication des acteurs externes peut jouer un rôle de levier dans la première phase d'une innovation simple, leur importance y sera accrue dans le cas d'une innovation complexe (Sørensen et Torfing, 2011). Enfin, la complexité implique un espace de l'innovation différent; espace physique tout d'abord (espace de travail favorable à la créativité individuelle et collective, voir Torugsa et Arundel (2016)), mais espace symbolique également puisque, comme nous l'avons vu, la complexité s'appuie sur des structures plus horizontales et sur un éventail de sources d'idées plus étendu. Ces éléments suggèrent que le rôle des réseaux d'acteurs change avec la complexité.

\subsection{Le rôle des réseaux d'acteurs sur l'innovation}

Un des espaces les plus importants dans la fabrique des innovations est peut-être l'espace social. En effet, le succès des innovations « ne repose pas sur la combinaisons des technologies et des systèmes, mais sur le fait de rassembler les gens » (Kinder, 2013 : 321). L'innovation est interstitielle : elle apparaît souvent dans les espaces situés entre les structures formelles, « où les individus peuvent échanger sans le fardeau des responsabilités formelles, des fonctions officielles et des règles » (Lewis et al., 2011; Lewis et Ricard, 2014). Le concept de réseaux d'acteurs désigne tant les réseaux officiels qu'officieux, formels et informels (Jones, Conway, et Steward, 1998), même si l'informalité peut également se pratiquer dans les espaces de la formalité. Autre élément de définition : les réseaux d'acteurs impliquent une multitude d'acteurs, ils ne sont donc pas de simples «partenariats » (souvent bi- ou trilatéraux) (Eggers et Singh, 2009).

Il peut paraître tautologique d'étudier le rôle des réseaux d'acteurs sur les innovations complexes, qui ont entre autres la particularité d'impliquer un grand nombre d'acteurs. En fait, le problème ne se pose pas car la simple implication d'acteurs organisés en réseaux ne signifie pas forcément que ces réseaux occupent un rôle plus important sur le processus d'innovation. Il faut donc distinguer l'ensemble des réseaux d'acteurs des « réseaux d'innovation », qui sont formés ou engendrés ad hoc en réponse à la perception au sein d'une organisation du besoin d'innover (Jones et al., 1998).

Encore une fois, les premières études à s'être intéressées au rôle des réseaux d'acteurs sur l'innovation viennent du secteur privé (Conway, 1995 ; Lundvall, 2010; Nelson, 1993). Les réseaux y sont modélisés comme des toiles de liens (ties) unissant des nœuds (nodes) qui agissent comme des leviers de l'innovation tant au niveau de l'organisation, du secteur d'activité ou même du pays. La littérature sur ce lien est vaste (Jones et al., 1998; Lewis et Ricard, 2014; Nelson, 1993). La sociologie a également accordé une importance prépondérante aux réseaux d'acteurs dans l'innovation. C'est le cas par exemple de la sociologie de l'action collective (M. Crozier et E. Friedberg, 1977; Reynaud, 1997) et, plus encore, de la théorie de la traduction (ou de l'acteur-réseau) (Akrich, Callon, et Latour, 2006), qui offre une manière de considérer les liens entre les modes de concertation d'un réseau et l'innovation, en mettant notamment l'emphase sur les mécanismes de conflit et de consensus. Les apports de la théorie de la traduction sont multiples. Elle élargit notamment le concept d'acteurs aux artefacts non humains (documents, machines, objets, etc.) qui prennent eux aussi part aux réseaux et qui participent au changement, dans le monde scientifique notamment (Gaglio, 2011). Par ailleurs, elle propose de considérer les innovations comme étant inscrites au sein de groupes sociaux ayant des intérêts antagonistes et mouvants (Dervaux, Pichault, et Renier, 2011 ; Fayolle, Lamine, et Chebbi, 2014). 


\subsection{Les réseaux d'acteurs et l'innovation publique}

Dans le secteur public, la littérature est moins avancée (Lewis et Ricard, 2014). Plusieurs auteurs ont tenté d'observer l'effet de certaines caractéristiques des réseaux sur l'innovation publique. Par exemple, Bekkers et al. (2013); ou encore Huijboom (2010), montrent qu'une certaine hétérogénéité des acteurs d'un réseau (formations, expériences, etc.) serait bénéfique pour l'innovation publique.

Mais l'importance d'autres caractéristiques de réseaux sur les processus d'innovation publique reste encore peu traitée. Dans cette partie, nous résumerons les apports, mais aussi les manques de la littérature sur le lien entre les réseaux et l'innovation publique en nous concentrons sur trois caractéristiques principalement : le degré d'ouverture-fermeture des réseaux, la qualité des liens et la centralité des acteurs.

L'ouverture des réseaux

Tout d'abord, les réseaux présentent des degrés d'ouverture variables vis-à-vis des acteurs et des réseaux tiers. En se basant sur les travaux de Bourdieu (2010), Huijboom (2010) explique que les réseaux sont sélectifs dans leur intégration de nouveaux membres dans le groupe, ils ont une propension à se fermer et s'homogénéiser.

Globalement la littérature insiste sur le rôle négatif de la fermeture du réseau sur l'innovation publique. En effet, cette fermeture agirait comme un frein en réduisant la communication et l'apport d'idées extérieures ou encore en installant un certain conformisme peu propice à l'innovation (Newman, Raine, et Skelcher, 2001 ; Teske et Schneider, 1994).

Dans la littérature sur le secteur privé, il a été montré que la fermeture du réseau pouvait aussi agir comme un levier sur l'innovation si celle-ci renforce les valeurs internes du groupe. Dans ce cas, les réseaux deviennent plus perméables à l'adoption d'innovations conformes à leurs valeurs dominantes. (Van der Vlies et Maas, 2009). Aucune étude ne montre cet effet levier de la fermeture dans le secteur public. C'est l'objet de notre première question.

\section{Question 1 : La fermeture d'un réseau peut-elle aussi servir de levier à l'innovation publique?}

\section{La qualité de liens entre les acteurs}

D'autres recherches se sont intéressées à l'effet sur l'innovation de la qualité des liens qui unissent les acteurs d'un réseau. Le concept de liens a été conceptualisé notamment par Granovetter (1973). Pour lui, les acteurs d'un réseau peuvent entretenir une relation forte et proche (homophile ou strong ties) : ils se connaissent bien, échangent des ressources et se font confiance. À l'inverse, deux acteurs peuvent se connaître malgré la distance symbolique (de milieux, d'institutions, etc.) qui les sépare et entretenir une relation réservée. On parle alors de lien faible (hétérophiles ou weak ties). Dans la littérature sur l'innovation dans le secteur privé, on souligne tant les avantages des liens faibles que des liens forts. Tandis que certains auteurs montrent les atouts d'un réseau composé d'une multitude de liens faibles, créant des « ponts » entre des univers potentiellement très différents, et mettant à disposition plus de ressources, d'informations et d'idées (Granovetter, 1973; Kaasa, 2009); d'autres de développer que quelques liens forts seulement (vecteurs de confiance) suffisent à la prise de risque si chère à l'innovation (Hulsink, Elfring, et Stam, 2008). Dans le cas de l'innovation publique, Huijboom (2010) montre que la force du lien peut avoir tour à tour un effet positif et négatif sur le processus d'innovation selon les phases du projet et de l'incertitude. Dans 
les phases où les barrières organisationnelles doivent être dépassées et que l'incertitude est grande, comme lors de la sélection des solutions (Sørensen et Torfing, 2011), l'existence de liens forts entre les nœuds est préférable. Les liens faibles, à l'inverse serviront davantage lors des phases de diffusion. Cela dit, les innovations complexes montrent un déroulement irrégulier et une forte incertitude tout au long du projet. C'est ce qui nous amène à la deuxième question.

\section{Question 2 : Comment agissent les liens forts et les liens faibles lorsque l'innovation est particulièrement complexe?}

\section{La centralité des acteurs et l'innovation publique}

Les acteurs n'occupent pas tous la même importance au sein d'un réseau. Certains sont dits centraux parce qu'ils sont perçus comme essentiels pour le projet par les autres acteurs (on parle de in-degree centrality ou de centralité de degré) mais aussi parce qu'ils sont des ponts entre des groupes peu connectés (betwenness centrality ou centralité d'intermédiarité) (Lacroix, 2003; Wasserman et Faust, 1994). Contre-intuitivement, le niveau hiérarchique est beaucoup moins déterminant dans la centralité d'un acteur que son importance stratégique (Considine, Lewis, et Alexander, 2009). Dans le secteur privé, ces intermédiaires permettent une accélération de la diffusion des idées (Burt, 2004) et peuvent créer du consensus. Dans le public, certaines recherches montrent que même s'ils peuvent jouer un rôle de levier, ces acteurs centraux (parfois nommés brokers (Lewis et Ricard, 2014) ou entrepreneurs institutionnels (Touati et al., 2016)) sont aussi capables de freiner puissamment l'innovation en utilisant à son encontre le pouvoir que leur position leur confère (Huijboom, 2010), pouvoir marginal sécant au sens de Crozier et Friedberg (1977). Dans le cas d'innovation complexes, la transversalité et le poids des frontières institutionnelles sont accrus. Tout laisse à penser que les acteurs centraux occupent une place encore plus importante. C'est ce que soulève notre troisième question.

Question 3 : Quel rôle occupent les acteurs centraux lorsque l'innovation est complexe et donc particulièrement confrontée aux barrières institutionnelles?

\section{Méthode et terrain de recherche}

\subsection{Méthodologie}

Afin d'explorer le rôle des réseaux d'acteurs dans une innovation publique complexe nous mobilisons une approche qualitative basée sur un cas exemplaire (Yin, 2011). Il s'agit en l'occurrence le projet du vote électronique (VE) dans le canton de Genève, depuis son début en 2001 jusqu'à aujourd'hui, à partir d'une collecte de données réalisée en 2016. Pour les besoins de l'étude, nous avons rencontré un responsable de la chancellerie de Genève qui nous a aiguillés vers quelques personnes-clés du projet. Au total nous avons conduit des entretiens semi-directifs d'environ une heure auprès de six acteurs importants entre septembre et décembre 2016. Même si ce genre de projet implique une multitude d'acteurs et d'organisations, l'équipe «noyau » du projet, au sein de la chancellerie, est assez réduite. C'est pourquoi le nombre d'entretiens, même s'il est relativement limité, suffit 
à raisonnablement couvrir les perceptions des acteurs principaux de l'équipe chargée du projet. Trois de ces acteurs font partie de l'équipe initiale de projet, et les trois autres de la nouvelle équipe (le projet a connu un changement d'équipe pendant son déroulement). Une analyse documentaire sur le cas (sites internet, interventions publiques, articles de presse et même articles scientifiques (Chevallier, 2009; Chevallier, Warynski, et Sandoz, 2006)) a servi à étayer les informations collectées lors des entretiens. Le sujet étant encore politiquement sensible, les entretiens n'ont pas été enregistrés par crainte de voir les répondants s'enfermer dans un discours officiel. Pour comprendre leur perception sur les rôles qu'ont joués les réseaux d'acteurs, et pour ne pas faire surestimer ces influences par rapport aux autres éléments potentiels, le guide d'entretien commence par demander aux répondants de retracer l'historique du projet et leur propre rôle dans celui-ci. Dans un second temps nous leur demandions, pour les différentes phases qu'ils avaient esquissées, les principaux leviers et freins. Ces deux premiers axes nous ont permis de cerner si les répondants mentionnaient que telle ou telle phase avait été facilitée ou freinée par l'existence d'un lien entre plusieurs acteurs, et de quelle manière. Enfin, nous les interrogions plus directement sur le rôle du « réseau » dans ce projet. Les mécanismes avancés ont été retranscrits dans le logiciel Nvivo puis codées de manière ouverte. Ces mécanismes perçus par les acteurs ont ensuite été organisés à travers une grille d'analyse construite sur le phasage proposé par Eggers et Singh (2009). Pour chacune des quatre phases, l'idée est d'analyser le rôle que les répondants accordent aux réseaux d'acteur (facilitant ou freinant) ainsi qu'à leurs caractéristiques (degré d'ouverture, qualité des liens, centralité des acteurs, etc.). Nous avons fait le choix d'aborder les caractéristiques de l'innovation de manière générique, et non en mobilisant un cadre théorique comme celui proposé par la sociologie de la traduction par exemple. Cette dernière est surtout basée sur l'étude de la création dynamique des réseaux et des mécanismes de coordination d'intérêts contradictoires menant à l'innovation (Fayolle $e t$ $a l ., 2014)$. Nous souhaitions ici adopter une démarche plus large pour comprendre, en aval du projet, quelle importance les acteurs accordaient aux réseaux et à leurs caractéristiques.

\subsection{Le cas du vote électronique dans le canton de Genève}

\section{Présentation du projet}

En 2001, la chancellerie fédérale suisse (l'État-major du gouvernement fédéral et l'organe responsable des droits politiques) souhaite répondre au problème du vote des Suisses expatriés. Leur vote par correspondance (VPC) -par voie postale-, n'arrive souvent pas à destination avant la fin des scrutins. La chancellerie décide donc de se tourner vers le vote par internet, le vote électronique (VE), et encourage les initiatives cantonales qui vont dans ce sens. Alors dans une dynamique de numérisation et de centralisation des dépouillements des votes par correspondance (en Suisse, le vote par voie postale est généralisé depuis 1995), le canton de Genève, -par l'intermédiaire de sa propre chancellerie -, répond présent à l'appel lancé par la confédération (ainsi que deux autres cantons). L'ambition de la chancellerie genevoise est d'aller plus loin que la seule résolution du problème des votants expatriés : elle compte sur le VE pour provoquer un sursaut de participation chez les électeurs, tel que suscité lors de l'adoption du VPC. La chancellerie met donc sur pied une équipe de projet (que nous nommerons l'équipe initiale) et choisit de développer le système en interne au lieu de l'externaliser. S'ensuit une période de concrétisation aux niveaux techniques et organisationnels, dans laquelle l'équipe de projet 
doit faire face à une série d'écueils (blocages politiques, budgets, aléas informatiques, etc.). Même si le système de VE est assez rapidement fonctionnel, les multiples blocages politiques et juridiques, tant au niveau fédéral que cantonal, l'empêchent de se déployer tout de suite à l'ensemble des électeurs genevois (20 pourcents des votants au maximum, par canton, peuvent utiliser le VE pour les scrutins fédéraux, plafond plus tard étendu à 30 pourcents), et aux élections (au départ le VE n'est utilisé que pour les votations ${ }^{2}$ ). Techniquement, le VE prend la forme d'une plateforme numérique sécurisée et centralisée, qui reçoit les votes, les anonymise et les stocke dans un serveur sécurisé jusqu'au dépouillement. En 2003, un des membres de l'équipe du projet, également maire d'une petite commune du canton saisit l'opportunité d'un scrutin communal pour utiliser le VE pour la première fois. Cet essai pilote est un succès (beaucoup de participation et pas de problème technique) : le système est inauguré et peut se répandre. En 2004, le VE est utilisé pour la première fois dans le cadre d'une votation sur un objet fédéral ${ }^{3}$. Malgré ces réussites, une épée de Damoclès pend au-dessus du projet : en cas de problème informatique ou de piratage, les votes sont susceptibles d'être perdus ou détournés. C'est pourquoi le conseil d'État (l'organe exécutif du canton) et la chancellerie fédérale se montrent prudents. La confédération, bien qu'étant une des instigatrices du projet, fixe des règles (comme le plafond des 30 pourcents). Pour donner plus de gages de sécurité et pour aller dans le sens des nouvelles réglementations sur le VE de plus en plus restrictives, le système passe en 2015 à un dispositif de vérifiabilité individuelle dans lequel les électeurs sont en mesure de suivre leur vote grâce à un système de codes personnels. En 2009, le chancelier cède sa place, et en 2013 l'équipe de projet initiale est remplacée par une nouvelle équipe.

En parallèle, les responsables du projet genevois imaginent «vendre » la « solution genevoise » aux autres cantons non impliqués dans la phase test, principalement pour abaisser les coûts d'exploitation. Ainsi, le système genevois de VE est « vendu » pour la première fois en 2009 à un autre canton (Bâle-Ville) et héberge aujourd'hui une partie des votes de cinq autres cantons suisses, malgré la présence sur le marché d'autres fournisseurs (privés) de solution de VE.

Au niveau des résultats globaux de cette politique, les statistiques de participation par mode de vote ${ }^{4}$ mis à jour en octobre 2016, montrent que le VE représente aujourd'hui environ $25 \%$ des votes au niveau cantonal ${ }^{5}$, alors que le VPC $70 \%$, et le vote aux urnes $5 \%$. Le VE prend de l'ampleur en tant que mode de vote. Toutefois, rien ne dit que le VE a réellement provoqué une diminution de l'abstention en proposant des moyens alternatifs de vote, comme le prévoyait la politique publique. En 2013, un rapport établi par la commission d'évaluation des politiques publiques quant à l'impact de l'introduction du VE, montrait que celui-ci avait davantage entraîné un glissement des autres modes de scrutin vers lui, sans pour autant impacter négativement l'abstention ${ }^{6}$.

\footnotetext{
2 En Suisse, les votations désignent les scrutins portant sur l'adoption ou le rejet de divers objets soumis au peuple par d'autres électeurs (initiatives populaires) ou par les autorités (référendum). On les distingue des élections qui elles portent sur le choix des représentants.

${ }^{3}$ Objet sur lequel tous les cantons suisses sont amenés à se prononcer

${ }^{4}$ Ces statistiques, menées par l'Office Cantonale de la Statistique, sont consultables ici : http://www. ge.ch/statistique/domaines/17/17_03/tableaux.asp\#11

5 Électeurs du canton et Suisses de l'étranger

${ }^{6}$ À consulter sur cette page: http://ge.ch/vote-electronique/media/site_vote-electronique/files/imce/ doc_rapports/cepp-rapport.pdf
} 


\section{Encadré 1 : Les grandes dates du projet}

2001 : lancement de l'appel à projet de la confédération. Genève, via sa chancellerie, accepte le défi et monte une équipe de projet.

2003 : Vote pilote dans une commune du canton. Le système est fonctionnel mais son usage n'est pas encore normalisé.

2004 : Utilisation dans le cadre d'un vote sur un objet fédéral.

2009 : Un autre canton l'adopte. Le chancelier est remplacé.

2013 : L'équipe de projet initiale se dissout, une nouvelle équipe de projet la remplace.

2015 : Passage à un système de vérifiabilité individuel. Le système peut être diffusé et utilisé à plus grande échelle, même si une norme plafond d'utilisation subsiste.

2016 : De nouveaux cantons commencent à adopter le système genevois.

\section{Une innovation complexe}

Ce long projet a donc démarré en 2001 et se diffuse aujourd'hui au reste des électeurs du canton, mais aussi aux autres cantons suisses. La «solution genevoise » est éminemment complexe. Technologiquement complexe tout d'abord : le nouveau service doit être avantageux pour les usagers malgré les contraintes sécuritaires. Un répondant va jusqu'à dire que «la complexité technique du projet crée un décalage avec les politiciens qui n'y comprennent rien ». Par ailleurs, le VE est multidimensionnel : il modifie concrètement la manière de voter des électeurs, et donc la manière de mener une campagne politique, et il oblige l'administration à se réorganiser. «C'est complexe car le nombre de dimensions à traiter est énorme : politique, technologique, etc.» affirme un de nos interviewés. C'est aussi une innovation conceptuelle parce qu'elle propose de reconsidérer l'usage des droits politiques à l'aune des technologies nouvelles. Par ailleurs, la mise sur pied du VE est transversale : en choisissant une « solution nationale » la chancellerie a dû faire appel aux divers services de l'État (notamment informatiques) pour le développement et la gestion du système. Le projet du VE est multi-acteurs puisque l'équipe de projet a dû concilier les objectifs, les vétos et les intérêts de la confédération, des organes exécutifs et législatifs cantonaux, des usagers finaux, des partis politiques, des autres cantons et des responsables politiques locaux, -notamment les maires-, premiers utilisateurs du système pour les élections locales. Enfin, le VE est un projet extrêmement risqué dans un milieu où la prudence est de mise : les droits politiques.

\section{Résultats}

Dans cette partie, nous présenterons la perception des répondants relative au rôle des réseaux sur le projet, de manière chronologique, et ce en suivant le phasage proposé par Eggers et Singh (2009) : l'émergence des idées, la phase de sélection, la mise en œuvre et la diffusion.

\section{Phase 1 : L'émergence de l'idée du vote électronique}

Selon les répondants, deux éléments essentiels sont susceptibles d'expliquer l'émergence de l'idée du VE. Le premier est l'inscription du projet dans une dynamique plus longue de numérisation des processus. Le canton aurait amorcé de nombreux projets de ce type dans 
plusieurs domaines et ce, grâce à son «dynamisme » et sa culture perçue comme plutôt centraliste. Le deuxième argument avancé par les répondants pour expliquer l'émergence du projet est quasi-unanimement le rôle du chancelier ${ }^{7}$ de l'époque (à la tête du service stratégique de l'administration cantonale). Cet homme est décrit tour à tour comme «un visionnaire », « un leader », « un fonceur » ou un homme possédant un fort caractère. C'est lui qui aurait saisi l'appel de la chancellerie fédérale et mis sur pied une équipe de projet qu'il va soutenir tout du long auprès de l'exécutif cantonal. Le rôle qu'occupe ce chancelier dans l'émergence du projet est fondamental pour nos répondants, au point qu'il semble l'incarner. Dans cette phase, le rôle des réseaux d'acteurs est assez peu évoqué.

\section{Phase 2 : La sélection des solutions}

À la suite de la décision du chancelier de relever le défi du VE, celui-ci monte une équipe de projet, composée d'une demi-douzaine de collaborateurs (l'équipe « initiale » dont trois de nos répondants font partie). Celle-ci est chargée de développer le système technique et de le mettre en œuvre. Cette étape est décrite comme relativement courte par nos répondants. Assez rapidement, l'équipe imagine les modalités techniques du VE qui sont plus ou moins celles d'aujourd'hui. En 2003 a lieu le premier scrutin VE dans une petite commune du canton dont le maire est également membre de l'équipe initiale. Le succès de cette phase est facilité, selon les répondants, par les caractéristiques de l'équipe. Ses membres auraient des parcours variés, des formations différentes et partageraient une culture de l'innovation, c'est-à-dire « le courage de prendre des risques » tout en gardant un certain « pragmatisme ». Les réseaux d'acteurs sont évoqués explicitement pour la première fois dans cette phase. C'est notamment le cas du « réseau des maires » : le maire de la commune pilote, également membre de l'équipe, dispose d'un grand capital sympathie et de plusieurs relations auprès de nombreux autres maires du canton (qu'il nomme « collègues »), parfois aussi députés cantonaux. Ceci fut un grand avantage pour le projet selon nos interlocuteurs, et ce pour trois raisons. Tout d'abord, parce que l'équipe a eu la capacité de sonder et d'anticiper les potentielles résistances des acteurs politiques (maires et députés-maires); ensuite parce qu'il a été dès lors possible d'identifier des « ambassadeurs », adjuvants capables de soutenir le VE auprès de leurs réseaux respectifs ; enfin parce que le projet a capitalisé sur la « confiance » (ce mot revient beaucoup pour décrire les relations entre maires) que les maires accordent à leur confrère. Si celui-ci utilise le VE dans sa propre commune, c'est qu'il doit être sûr : « ce n'est pas une lubie de technocrates » explique un de nos répondants. Notons que les maires peuvent utiliser le VE au niveau de leurs communes, mais pour une utilisation cantonale ils ne sont pas partie prenante de la décision, qui revient au parlement cantonal.

\section{Phase 3 : La mise en œuvre du système}

Le scrutin pilote semble être un tournant puisque l'après 2003 est beaucoup plus marqué par des activités de communication et de persuasion que par des réflexions sur l'applicabilité du VE selon nos répondants. Il semble que l'on ait entamé à ce moment la phase de mise en œuvre. Contrairement à la sélection des solutions, cette phase est vue comme extrêmement longue et laborieuse. Et pour cause, de nombreux « blocages juri-

\footnotetext{
${ }^{7}$ Le chancelier est le plus haut fonctionnaire d'une administration cantonale, il dirige le service stratégique et transversal appelé « chancellerie »
} 
diques et politiques » freinent le projet. Parmi eux, les répondants énoncent la «peur» des « députés-maires de voir leur mode d'élection bouleversé et de devoir être présents sur les réseaux sociaux », c'est-à-dire adopter de nouveaux modes de communication. Ce blocage expliquerait la relative difficulté qu'éprouve le VE à s'appliquer aux élections, et non plus seulement aux votations : il aurait fallu environ treize ans pour normaliser le VE dans le cadre des élections. D'autres péripéties vont ralentir, voire bloquer le projet (retrait du soutien du gouvernement, aléas techniques, etc...). Tous ces imprévus sont, pour nos répondants, fortement liés à la haute teneur en risque du projet perçue par l'ensemble des acteurs, qui souvent préfèrent la prudence à l'audace.

Cette phase de mise en œuvre est encore portée par l'équipe de base. Celle-ci s'appuie, selon nos interlocuteurs, sur deux types de réseaux. Le premier est le réseau des maires présenté plus haut. Le second est ce que l'on pourrait baptiser le « réseau des partis ». Un de nos répondants qui a pris part à l'équipe de projet initiale avance que chacun des membres de cette équipe entretient des « accointances personnelles avec des partis politiques différents ». Ces liens auraient grandement facilité les opérations destinées à convaincre chacun des partis. En fonction du parti qu'il convenait de convaincre, le représentant du projet changeait. Chaque membre de l'équipe initiale a donc mobilisé son propre réseau capitalisé à travers leur expérience politique, dans l'idée de convaincre les partis et de faciliter la mise en œuvre au parlement notamment.

\section{Phase 4 : La diffusion de la « solution genevoise »}

Les phases de mise en œuvre et de diffusion se sont développées parallèlement : alors que l'on cherchait encore à convaincre les partis-prenantes des avantages du VE au sein du canton, on commençait aussi à le diffuser aux autres cantons demandeurs. Notons que le projet a subi un deuxième «tournant » en 2013. Cette année est marquée par le remplacement de plusieurs des membres de l'équipe de projet, dans un contexte affecté par des mauvaises publicités (problèmes techniques notamment) qui ont provoqué quelques retraits de cantons tiers. La relève est donc assurée par une nouvelle équipe de projet, soutenue par une nouvelle chancelière en poste depuis 2009. La transition est décrite comme « difficile » par les membres de la nouvelle équipe, qui évoquent notamment la forte personnification du VE autour des membres de l'ancienne équipe. Cette transition est aussi en partie perçue comme bénéfique car elle aurait permis de désamorcer des blocages en partie liés à des problèmes interpersonnels charriés par les membres de l'ancienne équipe. La nouvelle équipe serait plus ouverte au compromis car plus flexible avec les principes de base du VE. «Quand le projet n'est pas votre bébé, vous êtes plus ouvert à la critique et plus flexible » explique un de nos répondants de la nouvelle équipe.

Les réseaux d'acteurs mentionnés par nos interlocuteurs ne sont plus les mêmes. En effet, les répondants de la nouvelle équipe évoquent comme leviers dans cette phase les bonnes relations institutionnelles qu'ils entretiennent en interne (notamment avec les services techniques) et en externes (avec les autres cantons). Or ces relations sont canalisées par des réseaux relativement formels. C'est le cas pour les liens entre l'équipe de projet et le service informatique, auparavant perçus comme litigieux et qui se sont normalisés après une « re-répartition formelle des tâches ». C'est aussi le cas des réseaux externes, notamment le réseau « des cantons » autour du VE en Suisse. Ce réseau, fondé par la confédération, permet au projet selon certains répondants, de trouver des ancrages dans d'autres cantons. 
Selon nos interlocuteurs, la phase de diffusion s'appuie sur des réseaux plus institutionnels, gravitant moins autour des réseaux personnels des membres de l'équipe de projet. La lecture proposée par la nouvelle équipe serait la suivante : les réseaux d'acteurs se formalisent et, conséquence souhaitée ou non, le projet s'affranchit des blocages liés aux tensions interindividuelles.

La figure 1 : résume les trois réseaux mis au jour lors de ces entretiens.

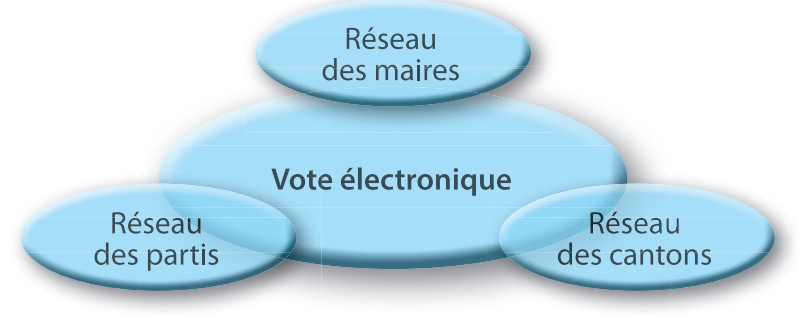

\section{4. Éléments de discussion}

Revenons sur nos résultats en mobilisant les apports de la littérature soulevés plus haut. Nous décrirons ici les caractéristiques des réseaux relevés : l'ouverture, la qualité des liens, la centralité des acteurs ainsi que leur composition. Ensuite, nous montrerons comment notre exemple fait ressortir l'idée selon laquelle les réseaux peuvent jouer un rôle différent en fonction des phases de l'innovation. Enfin nous ferons un bref retour la notion de complexité de l'innovation publique à la lumière de nos résultats.

\subsection{L'ouverture des réseaux}

De prime abord, il semble que la plupart des réseaux évoqués dans cette recherche soient relativement fermés au sens de Bourdieu (2010) et de Huijboom (2010), l'intégration de nouveaux membres et d'idées éloignées de leur valeur est a priori difficile. C'est notamment le cas des réseaux de partis et de maires. Toutefois, il est possible que cette ouverture change d'un parti à l'autre. Même s'il est difficile de trancher sur l'importance de cette fermeture et sur son rôle sur le processus, nous pouvons affirmer qu'un des grands atouts du projet réside dans la proximité qu'entretiennent les membres de l'équipe initiale avec des réseaux importants (partis politiques, politiciens, etc...). Dès lors, les obstacles liés aux difficultés de communications qu'impliquent ce genre de réseaux fermés (Newman et al., 2001 ; Teske et Schneider, 1994) sont contournés. Nous suggérons que si ces connivences personnelles n'existaient pas, la relative fermeture de ces réseaux aurait pu être un obstacle. C'est ce que nous montre notamment la difficile transition entre les deux équipes de projet.

\subsection{La qualité des liens}

À travers ce cas, on retrouve les différents types de lien présentés par Granovetter (1973). L'équipe de projet semble entretenir des liens forts (strong ties) avec le réseau des maires : les répondants évoquent régulièrement la « confiance » qui unit ses membres, par ailleurs habitués à communiquer ensemble. Le réseau des partis politiques ou encore 
celui des cantons, semblent unir des acteurs qui se connaissent peu et n'ont pas l'habitude de travailler ensemble. Les liens seraient plus faibles ici. Le fait que le réseau des maires (liens forts) soit mobilisé lors de la sélection des solutions et la mise en œuvre,-des phases à haute incertitude-, et que les réseaux à liens faibles, comme celui des cantons, le soient dans la diffusion, va dans le sens de Huijboom (2010).

\subsection{La centralité des acteurs}

Le concept de centralité des acteurs (Lacroix, 2003; Wasserman et Faust, 1994) est, lui, très visible dans notre exemple. C'est notamment le cas de l'ancien chancelier qui occupe une place de centralité de degré au sens de Wasserman et Faust (1994). Il incarne l'émergence du projet aux yeux des autres acteurs. Nous n'avons cependant que très peu d'informations sur ses liens avec d'autres réseaux. Deux explications pourraient être avancées à cela, toutes deux liées à la personnification de l'émergence du projet autour de la personnalité du chancelier. La première raison serait que les réseaux d'acteurs soient objectivement peu mobilisés dans la première phase puisque le caractère et les prérogatives du chancelier lui donnent les moyens de la porter seul. La seconde vient peut-être du fait que nos répondants, même ceux qui suivent le projet depuis le début, n'ont pas connaissance des réseaux que le chancelier a mobilisés. On peut raisonnablement supposer par exemple que des liens entre des membres de la chancellerie fédérale et lui-même aient pu faciliter le fait que Genève relève le défi.

Les membres de l'équipe initiale ont aussi des rôles de centralité de degré en ce qu'ils paraissent essentiels pour les répondants. Mais les membres de cette équipe ont aussi un rôle de centralité d'intermédiarité : ils sont des ponts entre le projet et leurs propres réseaux. Ces acteurs sont donc des interfaces puisqu'ils servent au projet à trouver écho dans les différents réseaux d'acteurs, tout en permettant aussi à leurs propres réseaux d'être représentés dans les négociations. Le rôle de cette centralité est globalement vu comme positif pour le VE, et se rapproche du concept d'entrepreneur institutionnel (Touati et al., 2016). Pour les répondants, le fait que les membres de l'équipe initiale aient accès par le biais de brokers à des réseaux importants comme celui des maires ou des partis est un levier. Mais il se peut que ce soit cette centralité qui ait contribué aussi aux blocages observés avant 2013 : la sur-centralité de quelques acteurs aurait cristallisé certaines appréhensions à l'encontre de ceux-là. Cette idée d'une influence négative de la centralité rejoint celle de Huijboom (2010).

\subsection{La composition des réseaux}

Comme nous l'avons vu, les membres de l'équipe initiale disposent tous d'un parcours et d'une formation différente. Leurs réseaux aussi sont divers dans leur composition. Par exemple les maires sont des élus qui présentent toutes sortes de profils, pratiquent tous types de professions ${ }^{8}$. Il y a donc une certaine hétérogénéité, une diversité des profils comme décrit par Bekkers et al. (2013), Huijboom (2010) et (Kinder, 2013). Ceci est perçu comme un levier, en accord avec la littérature.

\footnotetext{
${ }^{8}$ Les élus maires ne sont souvent pas employés à plein temps. Ils peuvent exercer ainsi leur métier de base.
} 


\subsection{Un rôle des réseaux sur l'innovation différent selon les phases}

Entre les relations formalisées et les structures officielles, les réseaux d'acteurs, jouent donc un rôle majeur dans la conduite d'une innovation publique, en freinant ou au contraire en catalysant le projet, en fonction de leurs caractéristiques. Ce que fait ressortir notre exemple, c'est l'idée que les caractéristiques de réseaux sur lesquelles s'appuient les innovations changent à chaque phase de développement.

Dans un sens, ceci corrobore les travaux de Sørensen et Torfing (2011) qui, eux s'intéressent aux phases de l'innovation, mais du point de vue de la collaboration.

Nos résultats ne mettent pas tellement en exergue le rôle du réseau d'acteurs au stade de l'émergence des idées (contrairement à Sørensen et Torfing (2011)). Nous l'avons vu, le réseau a très peu été invoqué à ce stade par nos répondants, qui semblaient plus donner le mérite de cette phase à la figure particulière du chancelier de l'époque. La phase de sélection des solutions est, elle, catalysée par les réseaux d'acteurs. Les réseaux d'acteurs facilitent l'accès à d'autres expertises et à de nouvelles ressources pour les innovateurs. Ils concourent aussi au compromis en fournissant des clefs de lectures aux innovateurs sur les potentiels vétos et intérêts à prendre en compte. Lors de la phase de mise en œuvre, les réseaux d'acteurs sont aussi présents dans notre cas sous plusieurs formes. Ils facilitent la mise en œuvre en capitalisant sur la confiance entre les membres pour se faire accepter et pour réduire leur incertitude. La phase de diffusion semble notamment s'articuler autour du réseau des cantons. Les réseaux servent ici de vecteurs de diffusion en permettant aux tiers d'obtenir plus facilement des retours d'expérience sur les amorces du projet. Dans ce cas le réseau principalement utilisé semble être plutôt formel et ses liens relativement faibles : la diffusion est large.

Nos résultats nous amènent donc à voir le rôle des réseaux d'acteurs sur l'innovation, non pas de manière constante, mais plutôt de façon différente pour chacune des phases du processus. Or, l'enchaînement de ces phases est particulièrement troublé lorsque l'innovation est complexe. Notre exemple montre une certaine irrégularité dans son déroulement. Certaines phases se chevauchent. C'est le particulièrement le cas des phases de mise en œuvre et de diffusion qui se développent en parallèle. Cette seconde idée rejoint les travaux de Eggers et Singh (2009).

\subsection{La perception de la complexité dans ce projet}

Nous l'avons vu, le projet du VE est multidimensionnel et multi-acteurs, il est complexe. Cependant il est également très risqué : le moindre échec conduirait à sa mise au ban. Cette notion de risque élevé est perçue par tous comme une dimension complexifiant grandement le projet. Les personnes à convaincre sont plus réticentes lorsqu'il y a un risque avéré, particulièrement les responsables politiques, les résistances sont plus vigoureuses et le travail est plus sollicitant. Ceci constitue une piste pour la littérature qui, à notre connaissance, n'a pas encore vraiment intégré le degré de risque perçu dans la définition d'une innovation publique complexe. 


\section{Conclusion}

Cette étude s'intéresse donc au rôle des réseaux d'acteurs dans le déroulement d'une innovation publique particulièrement complexe, à partir de la perception d'acteurs-clés . L'idée étant d'ajouter une pierre à l'édifice de la littérature sur l'innovation publique qui doit s'autonomiser et s'affranchir de l'importation brute des concepts du privé. En fait l'innovation publique serait singulièrement complexe : le nombre d'acteurs et de dimensions qu'elle implique modifie considérablement la manière d'appréhender les processus d'innovation et leurs «ingrédients ». Nous l'avons vu : les réseaux d'acteurs s'immiscent dans les processus d'innovation, mais cette interférence évolue avec la complexité. Cette étude apporte quelques éléments de réponses aux questions soulevées plus haut.

1.Même si nous disposons de relativement peu d'information sur la fermeture des réseaux dans notre exemple, il semble que la fermeture ne soit pas si freinante pour un projet, si les équipes de projets ont des accès privilégiés à ces réseaux fermés.

2.La confiance est un maître-mot dans ce projet. Les liens forts sont largement mobilisés afin de réduire l'incertitude que drague ce projet. Cet exemple suggère que les projets complexes doivent beaucoup compter sur ces réseaux à liens forts.

3. La centralité est essentielle dans notre exemple. Elle est un levier dans plusieurs de ses phases. Cependant elle conduit à la personnification du projet et peut agir comme un frein si ces personnes centrales cristallisent des conflits ou si leur remplacement n'est pas assuré correctement.

Notre étude de cas exemplaire apporte donc des éléments de réponses à ces questions, mais il convient d'en préciser les limites, dont la plupart sont inhérentes à ce type d'approche (étude de cas exemplaire unique) comme notamment l'absence de validité externe. On peut aussi déplorer le faible nombre d'entretiens, même si les acteurs entendus jouent tous (ou ont joué) un rôle central dans ce projet. Une étude ultérieure pourrait notamment prendre en compte les perceptions d'autres acteurs, extérieurs à l'équipe de projet, mais tout de même impliqués.

Malgré ces limites, deux nouvelles idées sont ressorties de notre exemple. Tout d'abord, notre cas présente une irrégularité dans son cheminement et un rôle inconstant des réseaux sur chacune des phases. Sans pouvoir généraliser, cet élément de discussion nous incite à considérer le rôle des réseaux, et plus généralement de tous les antécédents de l'innovation publique, de manière dynamique. Un antécédent important lors d'une phase de l'innovation ne le sera peut-être pas lors d'une autre phase. Semblablement, le degré de complexité même est susceptible d'évoluer au fur et à mesure du projet, tant les dimensions de l'innovation et le répertoire d'acteurs impliqués changent. Les recherches futures sur l'innovation publique doivent de toute évidence adopter cette approche de la non-linéarité des processus d'innovation. Le second point important soulevé par notre exemple relève de la notion de risque dans la complexité. Les recherches futures gagneraient à intégrer le risque, ou tout du moins le risque perçu d'une innovation, dans l'évaluation de sa complexité. 


\section{Bibliographie}

Akrich M., Callon M. et Latour, B., 2006. Sociologie de la traduction : textes fondateurs. École des mines de Paris, Paris.

Alexander D., Lewis J. M. and Considine M., 2011. How Politicians and Bureaucrats Network: A Comparison across Governments. Public Administration 89 (4), 1274-1292.

Armbrustera H., Bikfalvib A., Kinkela S. and Laya, G., 2008. Organizational Innovation: The Challenge of Measuring Non-Technical Innovation in Large-Scale Surveys. Technovation 28, 644-657.

Bekkers V. J. J. M., Tummers L. and Vooberg W., 2013. From Public Innovation to Social Innovation in the Public Sector: A Literature Review of Relevant Drivers and Barriers. Paper presented at the EGPA 2013 Conference, Rotterdam.

Boukamel O. and Emery Y. 2017. Evolution of Organizational Ambidexterity in the Public Sector and Current Challenges of Innovation Capabilities. The Innovation Journal: The Public Sector Innovation Journal 22 (2), article 2.

Bourdieu P., 2010. The Forms of Capital (1986) In I. Szeman and T. Kaposy (Eds.), Cultural Theory: An Anthology, Wiley, chichester, 548.

Burt R., 2004. Structural Holes and Good Ideas. American Journal of Sociology 110 (2), 349-399.

Camisón C. and Villar-López A., 2014. Organizational Innovation as an Enabler of Technological Innovation Capabilities and Firm Performance. Journal of Business Research 67 (1), 2891-2902.

Chesbrough H. W., 2003. Open Innovation: The New Imperative for Creating and Profiting from Technology. Harvard Business Press, Boston.

Chevallier M., 2009. Internet Voting, Turnout and Deliberation: A Study. Electronic Journal of e-Government 7 (1), 29-44.

Chevallier M., Warynski M. and Sandoz A. 2006. Success Factors of Geneva's e-Voting System. The Electronic Journal of e-Government 4 (2), 55-62.

Christensen T. and Lægreid P., 2016. Organizational Innovations and Multiple Forms of Accountability in the post-New Public Management Era In J. Torfing and P. Triantafillou (Eds.), Enhancing Public Innovation by Transforming Public Governance, Cambridge University Press, Cambridge, 351.

Considine M., Lewis J. M. and Alexander, D., 2009. Networks, Innovation and Public Policy : Politicians, Bureaucrats and the Pathways to Change Inside Government, Palgrave Macmillan, Basingstok.

Conway S., 1995. Informal Boundary-Spanning Communication in the Innovation Process: an Empirical Study. Technology Analysis \& Strategic Management 7 (3), 327-342.

Crozier M. et Friedberg E., 1977. L'acteur et le système. Le Seuil, Paris.

De Vries H., Bekkers V. J. J. M. and Tummers, L., 2015. Innovation in the Public Sector: A Systematic Review and Future Research Agenda. Public Administration 94 (1), 146-166.

Dervaux A., Pichault F. et Renier, N., 2011. L'apport de la théorie de l'acteur-réseau à la professionnalisation de la GRH en milieu hospitalier. Journal d'économie médicale 29 (1), 73.

Dougherty D. and Dunne D.D., 2011. Organizing Ecologies of Complex Innovation. Organization Science 22 (5), 1214-1223

Eggers B. and Singh, S., 2009. The Public Innovators Playbook. Harvard Kennedy School of Government, Washington, DC.

Fayolle A., Lamine W. et Chebbi, H., 2014. Quel apport de la théorie de l'acteur-réseau pour appréhender la dynamique de construction du réseau entrepreneurial? Management international 19 (1), 158-176.

Fu W., Revilla Diez J. and Schiller, D., 2013. Interactive Learning, Informal Networks and Innovation: Evidence from Electronics Firm Survey in the Pearl River Delta, China. Research Policy 42 (3), 635-646.

Gaglio G., 2011. Sociologie de l'Innovation. PUF, Paris.

Goffin K. et Mitchell R., 2010. Innovation Management: Strategy and Implementation using the Pentathlon Framework, Second Édition. Palgrave Macmillan, London. 
Gopalakrishnan S. and Bierly P., 2001. Analyzing Innovation Adoption using a Knowledge-Based Approach. Journal of Engineering and Technology Management 18 (2), 107-130.

Gopalakrishnan S. and Damanpour F., 1994. Patterns of Generation and Adoption of Innovation in Organizations: Contingency Models of Innovation Attributes. Journal of Engineering and Technology Management 11 (2), 95-116.

Granovetter M. S., 1973. The Strength of Weak Ties. American Journal of Sociology 78 (6), 1360-1380.

Halvorsen T., Hauknes J., Miles I. and Rannveig R., 2005. On the Differences Between Public and Private Sector Innovation. Retrieved from http://unpan1.un.org/intradoc/groups/public/documents/apcity/unpan046809.pdf

Huijboom N., 2010. Joined up ICT Innovation in Government. Erasmus University, Rotterdam.

Hulsink W., Elfring T. and Stam W., 2008. The Locus of Innovation in Small and Medium-sized Firms: The Importance of Social Capital and Networking in Innovative Entrepreneurship. Retrieved from Rotterdam: https://papers.ssrn.com/sol3/papers.cfm?abstract_id=1170184

Jones O., Conway S. and Steward, F., 1998. Introduction: Social Interaction and Innovation Networks. International Journal of Innovation Management 2 (2), 123-136.

Kaasa, A., 2009. Effects of different dimensions of social capital on innovative activity: Evidence from Europe at the regional level. Technovation, 29 (3), 218-233.

Kattel R., 2015. What would Max Weber Say about Public-Sector Innovation? NISPAcee Journal of Public Administration and Policy 8 (1), 9-19.

Kattel R., Cepilovs A., Kalvet T., Lember V. and Tonurist P., 2016. Public Sector Innovation Indicators: Towards a New Evaluative Frameworks. Retrieved from www.lipse.org

Kay R. and Goldspink C., 2016. Public Sector Innovation: Why it's Different. Retrieved from http://www. companydirectors.com.au/

Kinder T., 2003. Mrs Miller Moves House: The Interoperability of Local Public Services in Europe. Journal of European Social Policy 13 (2), 141-157.

Kinder T., 2013. Innovation in an inter-organisational context In S. P. Osborne and L. Brown (Eds.), Handbook of Innovation in Public Services. Edward Edgar Publishing, Northampton MA.

Lacroix M., 2003. Littérature, analyse de réseaux et centralité : esquisse d'une théorisation du lien social concret en littérature. Recherches sociographiques 44 (3), 475-497.

Lewis J. M., Considine M. and Alexander D., 2011. Innovation Inside Government: The Importance of Networks In V. Bekkers J. Edelenbos and Steijn B. (Eds.), Innovation in the Public Sector: Linking Capacity and Leadership, Palgrave Macmillan, London, 107-133.

Lewis J. M. and Ricard L. M., 2014. Innovation Capacity in the Public Sector: Structures, Networks and Leadership. Retrieved from Rotterdam: http://www.lipse.org/

Lundvall B. A., 2010. National Systems of Innovation: Toward a Theory of Innovation and Interactive Learning. Anthem Press, London.

Michaux V., 2010. Innovations à l'interface entre institutions publiques, para-publiques et privées dans le cadre des politiques publiques préventives concertées : le cas de la prévention des licenciements pour raison de santé. Management \& Avenir 5 (35), 210-234.

Nelson R., 1993. National Innovation Systems: A Comparative Analysis. Oxford University Press, Oxford.

Newman J., Raine J. and Skelcher, C., 2001. Developments: Transforming Local Government: Innovation and Modernization. Public Money \& Management 21 (2), 61-68.

OECD/Eurostat, 2005. Manuel d'Oslo : Principes directeurs pour le recueil et l'interprétation des données sur l'innovation, $3^{\mathrm{e}}$ édition. OECD Publishing, Paris.

Osborne S. P. and Brown L., 2011. Handbook of Innovation in Public Services. Edward Edgar Publishing, Northampton.

Pelz D. C., 1985. Innovation Complexity and the Sequence of Innovating Stages. Knowledge: Creation, Diffusion and Utilization 6 (3), 261-291. 
Pollitt C. and Bouckaert G., 2011. Public Management Reform: a comparative analysis. Oxford University Press, Oxford.

Reynaud J.-D., 1997. Les règles du jeu. L'action collective et la régulation sociale. Armand Colin, Paris.

Rhodes M. L., 2013. Innovation in Complex Public Services Systems In S. P. Osborne and L. Brown (Eds.), Handbook of Innovation in Public Services, Edward Edgar Publishing, Northampton, 332-359.

Salge T.O. and Vera A., 2012. Benefiting from Public Sector Innovation: The Moderating Role of Customer and Learning Orientation. Public Administration Review 72 (4), 550-560.

Sørensen E. and Torfing J., 2011. Enhancing Collaborative Innovation in the Public Sector. Administration \& Society $43(8), 842-868$.

Teske, P., et Schneider, M., 1994. The Bureaucratic Entrepreneur: The Case of City Managers. Public Administration Review, 54 (4), 331-340.

Torugsa N. and Arundel A., 2016. Complexity of Innovation in the public sector: A workgroup-level analysis of related factors and outcomes. Public Management Review 18 (3), 392-416.

Touati N., Denis J.-L., Grenier C. and Smits P., 2016. Implementing Spaces to Favor the Emergence of Ecologies of Complex Innovation in the Public Sector: An Empirical Analysis. Administration \& Society, 1-28.

Van der Vlies R.D. and Maas G. J., 2009. A Social Capital Perspective to Innovation Management. Paper presented at the 26th International Symposium on Automation and Robotics in Construction.

Walker R.M., 2008. An Empirical Evaluation of Innovation Types and Organizational and Environmental Characteristics: Towards a Configuration Framework. Journal of Public Administration Research and Theory 18 (4), 591-615.

Wasserman S. and Faust K., 1994. Social Network Analysis: Methods and Applications, Cambridge University Press, Cambridge.

Windrum P., 2008. Innovation and Entrepreneurship in Public Services In P. Windrum and P. Koch (Eds.), Innovation in Public Sector Services, Edward Elgar, Cheltenham, 3-20.

Yin R. K., 2011. Applications of Case Study Research. SAGE Publications, London.

Zietsma C. and Lawrence T. B., 2010. Institutional Work in the Transformation of an Organizational Field: The Interplay of Boundary Work and Practice Work. Administrative Science Quarterly 55 (2), 189-221. 